\title{
Investigations of bulk and surface spin wave modes in FeNi multilayers by spin wave resonance
}

\author{
R. Kordecki ${ }^{\text {a }}$, R. Meckenstock ${ }^{\text {a }}$, J. Pelzl ${ }^{\text {a }}$, S. Nikitov ${ }^{b}$ and J.C. Lodder ${ }^{\mathrm{c}}$ \\ ${ }^{a}$ Inst. für Experimentalphysik, AG Festkörperspektroskopie, Ruhr-Universität, P.O. Box 1021 48, 4630 Bochum 1, Germany \\ ${ }^{b}$ Institute of Radio Engineering and Electronics, Academy of Sciences, Moscow, Russian Federation \\ ${ }^{c}$ MESA Research Institute, University of Twente, P.O. Box 217, 7500 AE Enschede, The Netherlands
}

\begin{abstract}
The SWR spectra of an FeNi multilayer have been studied experimentally and theoretically. Non-propagating surface spin wave modes as well as standing volume modes could be observed. Values of the surface anisotropy, the spin wave stiffness constant and the effective magnetisation have been deduced from the spectra. The results obtained by SWR and torque measurements are discussed.
\end{abstract}

\section{Introduction}

Spin wave resonance (SWR) is well established for investigations of magnetic parameters of single crystals and thin films, but has been used for magnetic multilayers only recently [1,2]. Surface and volume anisotropies and the spin wave stiffness constant of the samples are the most prominent parameters which can be studied by SWR. The surface anisotropy can lead to the appearance of surface spin wave modes in the SWR pattern. The excitation of such modes in thin magnetic films has been found in several experiments [3] and is explained by theoretical work [4]. The existence of surface modes in multilayered thin films has been shown recently in different SWR experiments $[5,6]$.

\section{Experiments}

The investigated magnetic multilayer was composed of alternating polycrystalline $\mathrm{Fe}$ and $\mathrm{Ni}$ layers with thicknesses of $d_{\mathrm{Fe}}=8 \mathrm{~nm}$ and $d_{\mathrm{Ni}}=10$ nm. $11 \mathrm{Ni}$ and $10 \mathrm{Fe}$ films have been deposited onto quartz crystal substrates by electron beam evaporation at room temperature in such a way

Correspondence to: Dr. Rolf Kordecki, Ruhr-Universität Bochum, Inst. für Experimentalphysik, AG Festkörperspektroskopie, NB 03/58, P.O. Box 102148, 4630 Bochum 1, Germany. Tel.: +49-234-7005864. E-mail: kordecki $@$ ruba.rz.ruhr-uni-bochum.de. that bottom and top layers of the structure were $\mathrm{Ni}$ films. The total thickness of the sample was $190 \mathrm{~nm}$. The multilayers have been characterised by in situ resistivity measurements, X-ray diffraction, and TEM analysis.

The torque curves of the films were measured at room temperature and at different values of the external magnetic field between 500 and 1400 $\mathrm{kA} / \mathrm{m}$. The magnet was mounted on a computer-controlled rotation stage. The direction of the external magnetic field was changed in the plane perpendicular to the sample surface. All measured torque curves show a strong uniaxial in-plane anisotropy of the samples. Even at the strongest applied magnetic field the multilayers were not fully saturated.

The SWR experiments have been carried out at 9.2 GHz. The spectrum of the parallel configuration (external magnetic field $B$ parallel to the sample surface) consists of only one peak with non-symmetrical shape centred at a magnetic field of about $70 \mathrm{mT}$ (fig. 1a). At intermediate field angles two well separated modes appear as shown in fig. $1 b$. In the perpendicular configuration $(B$ $\perp$ film plane) a full standing spin wave resonance pattern appears at resonance field values of about $2 \mathrm{~T}$ (fig. 1c).

\section{Results and discussion}

The measured spectra of the $\mathrm{Fe} / \mathrm{Ni}$ multilayer studied in this work can be explained most satis- 

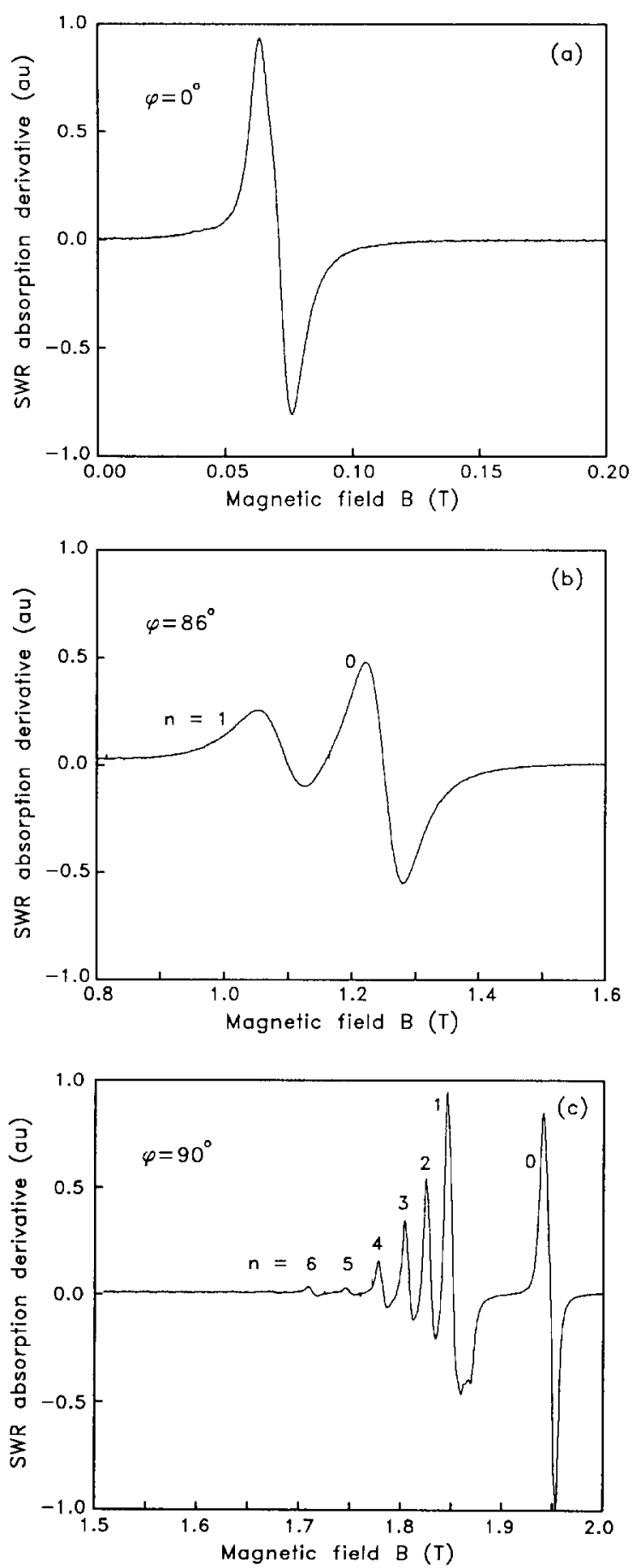

Fig. 1. Experimental spin wave resonance spectra of an FeNi multilayer $\left(d_{\mathrm{Fe}}=8 \mathrm{~nm}, d_{\mathrm{Ni}}=10 \mathrm{~nm}\right)$ for different angles $\varphi$ of the external magnetic field $B$. factorily on the assumption that spin waves extend across the whole multilayer with an average magnetisation. The derivation of averaged magnetic parameters of multilayered structures has been published elsewhere [1,7]. In the case of infinite strong magnetic coupling at the $\mathrm{FeNi}$ interfaces, the resonance frequencies of spin wave modes for the perpendicular configuration are given by

$\omega / \gamma_{\mathrm{eff}}=B_{\mathrm{res}}-\mu_{0} M_{\mathrm{eff}}+D_{\mathrm{eff}} q^{2} / \gamma_{\mathrm{eff}} \hbar$,

where $\omega$ is the microwave frequency, $\gamma_{\text {eff }}$ the effective gyromagnetic ratio, $B_{\text {res }}$ the resonance field, $D_{\text {eff }}$ the effective spin wave stiffness constant and $q$ the wave number of the corresponding spin wave mode, which can be real or imaginary. The effective magnetisation $M_{\text {eff }}$ is given by [1]

$M_{\mathrm{eff}}=\left\langle M^{2}\right\rangle /\langle M\rangle-2 K_{\mathrm{u}} / \mu_{0}\langle M\rangle$.

The average of a value $X$ is defined by $\langle X\rangle=$ $\left(d_{\mathrm{Fe}} X_{\mathrm{Fe}}+d_{\mathrm{Ni}} X_{\mathrm{Ni}}\right) /\left(d_{\mathrm{Fe}}+d_{\mathrm{Ni}}\right)$, where $X_{\mathrm{Fe} / \mathrm{Ni}}$ are the values of pure $\mathrm{Fe}$ and $\mathrm{Ni}$ and $d_{\mathrm{Fe} / \mathrm{Ni}}$ are the thicknesses of the individual layers. $M_{\text {eff }}$ includes in general a uniaxial anisotropy $K_{\mathrm{u}}$ [1].

To solve the problem of spin wave excitations in an external magnetic field one has to apply boundary conditions for the high frequency components of the magnetisation at the top and bottom surfaces [3]. The assumption of equal surface anisotropy $K_{\mathrm{s}}$ at the two surfaces of the sample leads to

$$
\begin{aligned}
& \tan (q L)=A_{\text {eff }} q K_{\mathrm{s}} /\left(K_{\mathrm{s}}^{2}-A_{\mathrm{eff}}^{2} q^{2}\right), \\
& \tanh \left(q_{\mathrm{s}} L\right)=A_{\mathrm{eff}} q_{\mathrm{s}} K_{\mathrm{s}} /\left(K_{\mathrm{s}}^{2}-A_{\mathrm{eff}}^{2} q_{\mathrm{s}}^{2}\right) .
\end{aligned}
$$

The first equation is valid for the standing volume spin wave modes and the second for the surface modes. $A_{\text {eff }}$ is the exchange constant of the multilayer, which is related to $D_{\text {eff }}$ by $D_{\text {eff }}=$ $2 A_{\text {eff }} /\langle M\rangle$.

With the measured resonance field positions of the surface (fig. 1c, $n=0$ ) and volume (fig. 1c, $n=1-6$ ) modes and by using the eqs. (1) and (3), we get the values of the surface anisotropy $K_{\mathrm{s}}=$ 
$-0.29 \times 10^{-3} \mathrm{~J} / \mathrm{m}^{2}$, the stiffness constant $D_{\text {eff }}=$ $2.6 \times 10^{-40} \mathrm{~J} \mathrm{~m}^{2}$ and the effective magnetisation $M_{\text {eff }}=1.21 \times 10^{6} \mathrm{~A} / \mathrm{m}$ of the multilayered structure. The wave number $q_{\mathrm{s}}$ of the surface mode is $q_{\mathrm{S}}=6.32 \times 10^{7} \mathrm{~m}^{-1}$ and the corresponding wavelength about $100 \mathrm{~nm}$, so this mode is localised at the surfaces of the sample, but extends over several periods of the multilayer.

The effective anisotropy field $H_{\text {an }}$ has been deduced from the torque curves by extrapolation of the first and second order Fourier coefficients to infinite field values. The measured value of the anisotropy field $H_{\text {an }}=1.00 \times 10^{6} \mathrm{~A} / \mathrm{m}$ is equal to the calculated value of $\langle M\rangle$. This result proves that there exists no additional perpendicular anisotropy $\left\langle K_{\mathrm{u}}\right\rangle$ in this multilayer and $H_{\text {an }}$ is only due to shape anisotropy.

The measured value of $M_{\text {eff }}$ is about $12 \%$ smaller compared to the value of $\left\langle M^{2}\right\rangle /\langle M\rangle$ calculated with the well-known magnetic parameters of $\mathrm{Fe}$ and $\mathrm{Ni}$. From the torque experiments $\left\langle K_{\mathrm{u}}\right\rangle$ was found to be zero, so the smaller value of $M_{\mathrm{eff}}$ can be only due to incomplete coupling at the FeNi interfaces. This result is supported by the value of the measured effective stiffness constant $D_{\text {eff }}$, which is about $40 \%$ smaller than the calculated one.

In order to get further proof of our interpretation of the surface mode we investigated the variation of the surface mode spectrum as a function of the angle $\varphi$ between $B$ and the plane of the film. The non-symmetrical shape of the resonance mode observed at $\varphi=0$ (fig. 1a) can be explained by the superposition of the nearly uniform resonance on the first volume spin wave mode, the two being very close in the parallel configuration. Due to the incomplete magnetic coupling at the FeNi interfaces the values of the resonance field position deviates from the value calculated by eqs. $(1,2)$, but $M_{\text {eff }}$ obtained from this field orientation is in agreement with the value which has been deduced at $\varphi=90^{\circ}$.

By changing $\varphi$ from zero to $90^{\circ}$ the asymmetric mode splits to two well-separated modes (fig. $1 b)$. The peak with the larger magnetic field value is attributed to the nearly uniform mode and the one with the lower value to the first spin wave mode. Further increase of the angle $\varphi$ leads to the transformation of the low field mode to the standing spin wave pattern. The high field mode transforms to the surface mode.

The angular dependence of the position of the spin wave modes has already been treated for the case of thin films [8-10]. It was shown that there exist critical angles $\varphi_{\text {crit }}$ of the external magnetic field where the uniform mode transforms to the surface mode and the standing spin waves appear. From our experiments we obtained a value of $\varphi_{\text {crit }}=86^{\circ}$, which is in good agreement with the calculated result.

\section{Conclusion}

In an $\mathrm{Fe} / \mathrm{Ni}$ multilayered structure we were able to observe non-propagating surface modes as well as standing volume spin wave modes. The surface mode is attributed to the presence of surface anisotropy at the surfaces of the structure. As the magnetic field changes from the parallel to the perpendicular configuration the transformation of the unpinned volume modes to the surface mode and the standing spin wave pattern could be observed. Comparison of the results obtained by SWR and torque measurements proves that there is no perpendicular anisotropy and incomplete interface coupling in the investigated FeNi multilayer.

The authors would like to thank Drs G. Dumpich and $H$. Mühlbauer for the sample preparation and the Deutsche Forschungsgemeinschaft (SFB 166) for financial support.

\section{References}

[1] R.P. van Stapele, F.J.A.M. Greidanus and J.W. Smits, J. Appl. Phys. 57 (1985) 1282.

[2] S.W. McKnight and C. Vittoria, Phys. Rev. B 36 (1987) 8574.

[3] Z. Frait and D. Fraitova, Spin wave resonance in metals, in: Spin Waves and Magnetic Excitation, part II, eds. A.S. Borovik-Romanov and S.K. Sinha (North-Holland, Amsterdam, 1988).

[4] H. Puszkarski, Prog. Surf. Sci. 9 (1979) 191.

[5] R. Kordecki, R. Meckenstock, J. Pelzl, H. Mühlbauer, G. Dumpich and S. Nikitov, J. Appl. Phys. 70 (1991) 6418 
[6] R. Krishnan, C. Sella, M. Kaabouchi, B.A. Acharya, S. Prasad and N. Ventkatramani, J. Magn. Magn. Mater. 104-107 (1992) 1822.

[7] A. Layadi and J.O. Artman, J. Magn. Magn. Mater. 92 (1990) 143.

[8] J.T. Yu, R.A. Turk and P.E. Wigen, Solid State Commun. 14 (1974) 283.
[9] J. Spalek and W. Schmidt, Solid State Commun. 16 (1975) 193.

[10] L.J. Maksymowicz and D. Sendorek, J. Magn. Magn. Mater. 37 (1983) 177. 